\title{
Doenças notificáveis segundo a forma de transmissão em um hospital de referência na Amazônia oriental brasileira
}

\author{
Notifiable diseases according to the form of transmission in a reference hospital in the \\ eastern Amazon of Brazil
}

\section{Enfermedades notificables según la forma de transmisión en un hospital de referencia en la Amazonia oriental brasileña}

Vanessa Pompeu Baia Miranda ${ }^{1 *}$, Gabriela de Cássia Oliveira dos Santos ${ }^{1}$, Thais Cristina Borges Farias $^{2}$, Silvia Helena Portilho de Barros ${ }^{1}$, Lourival Rodrigues Marsola ${ }^{2}$, Ariana Santana da Silva ${ }^{2}$, Marcela Emilly da Silva Pereira ${ }^{1}$, Jennifer Karen Ferreira Macena ${ }^{1}$, Danielle Saraiva Tuma dos Reis ${ }^{1}$.

\section{RESUMO}

Objetivo: Descrever dados clínico-epidemiológicos, a taxa de incidência e mortalidade em municípios das mesorregiões paraense relacionados às Doenças de Notificação Compulsória (DNC), ano 2018, segundo as doenças de transmissão respiratória, infecções transmitidas por insetos vetores, doenças relacionadas ao contato com coleções de água/solo e Infecções Sexualmente Transmissíveis. Métodos: Estudo descritivo, retrospectivo, transversal e quantitativo. População composta por notificações compulsórias de usuários confirmados, segundo critérios de inclusão preestabelecidos. Utilizado o banco de dados próprio do serviço de vigilância epidemiológica hospitalar onde o estudo ocorreu. Resultados: As DNC destaques foram Meningites, Tuberculose, Leishmaniose Visceral e Síndrome da imunodeficiência adquirida $\geq 13$ anos, esta última com predomínio na taxa de mortalidade. O sexo masculino prevaleceu, havendo notoriedade para lactantes, crianças e adultos jovens; municípios do Nordeste Paraense foram mais numerosos e insidiosos. Conclusão: Este estudo evidenciou DNC com grande visibilidade no estado do Pará, dentre as quais, as de transmissão respiratória. Resultados referentes ao perfil clínico-epidemiológico estavam intrinsecamente relacionados às características epidemiológicas de DNC prevalentes no estudo, já impactos socioambientais podem justificar a notoriedade à mesorregião Nordeste do estado. A Amazônia Oriental urge pesquisas epidemiológicas, que norteiem políticas públicas de saúde aos moldes das reais necessidades da população paraense.

Palavras-chave: Vigilância em saúde pública, Doença sexualmente transmissível, Notificação compulsória, Política pública.

\begin{abstract}
Objective: To describe clinical and epidemiological data, the incidence and mortality rate in municipalities of the Pará mesoregions related to Compulsory Notifiable Diseases (CND), year 2018, according to respiratory transmission diseases, infections transmitted by insect vectors, diseases related to contact with water/soil collections and Sexually Transmitted Infections. Methods: Descriptive, retrospective, transversal and quantitative study. Population consisting of compulsory notifications from confirmed users, according to preestablished inclusion criteria. The database of the hospital epidemiological surveillance service where the study took place was used. Results: The highlighted CNDs were Meningitis, Tuberculosis, Visceral Leishmaniasis and Acquired Immunodeficiency Syndrome $\geq 13$ years, the latter with a predominance in the mortality rate. The male gender prevailed, with notoriety for infants, children and young adults; municipalities in the Northeast of Pará were more numerous and insidious. Conclusion: This study evidenced CNDs with high visibility in the state of Pará, among which those of respiratory transmission. Results regarding the clinical-epidemiological profile were intrinsically related to the epidemiological characteristics of CNDs prevalent in the study, while socio-environmental impacts can justify the notoriety of the state's Northeast mesoregion. The Eastern Amazon urges epidemiological research to guide public health policies in line with the real needs of the Pará population.
\end{abstract}

Keywords: Public health surveillance, Sexually transmitted diseases, Compulsory notification, Public policy.

1 Universidade Federal do Pará, Belém - PA. *E-mail: enf.vanessabaia@gmail.com

2 Universitário João de Barros Barreto, Belém - PA. 


\section{RESUMEN}

Objetivo: Describir datos clínicos y epidemiológicos, la incidencia y tasa de mortalidad en municipios de las mesorregiones de Pará relacionadas con Enfermedades de Notificación Obligatoria (ENT), año 2018, según enfermedades de transmisión respiratoria, infecciones transmitidas por insectos vectores, enfermedades relacionadas con el contacto con el agua. / Colecciones de suelo e Infecciones de Transmisión Sexual. Métodos: Estudio descriptivo, retrospectivo, transversal y cuantitativo. Población compuesta por notificaciones obligatorias de usuarios confirmados, según criterios de inclusión preestablecidos. Se utilizó la base de datos del servicio de vigilancia epidemiológica hospitalaria donde se realizó el estudio. Resultados: Las ENT destacadas fueron Meningitis, Tuberculosis, Leishmaniasis Visceral y Síndrome de Inmunodeficiencia Adquirida $\geq 13$ años, este último con predominio en la tasa de mortalidad. Predominó el género masculino, con notoriedad para infantes, niños y adultos jóvenes; los municipios del noreste de Pará eran más numerosos e insidiosos. Conclusión: este estudio evidenció ENT con alta visibilidad en el estado de Pará, entre las que se encuentran las de transmisión respiratoria. Los resultados en cuanto al perfil clínico-epidemiológico se relacionaron intrínsecamente con las características epidemiológicas de las ENT prevalentes en el estudio, mientras que los impactos socioambientales pueden justificar la notoriedad de la mesorregión nororiental del estado. La Amazonía Oriental insta a la investigación epidemiológica para orientar las políticas de salud pública en consonancia con las necesidades reales de la población de Pará.

Palabras clave: Vigilancia de la salud pública, Enfermedad de transmisión sexual, Notificación obligatoria, Política pública.

\section{INTRODUÇÃO}

A Vigilância em Saúde envolve a coleta, consolidação, análise e disseminação dos dados relacionados aos eventos em saúde, de modo contínuo e sistemático, para planejar e implementar políticas públicas para proteção e promoção da saúde. Em contrapartida, as notificações de Doenças de Notificação Compulsória (DNC) são de comunicação obrigatória à autoridade de saúde, devido sua gravidade, magnitude, transcendência e capacidade de disseminação do agente causador, além de potencial para surtos e epidemias (MINISTÉRIO DA SAÚDE, 2020; SEGURADO AC, et al., 2016).

As doenças infecciosas de interesse para a saúde pública segundo o impacto na morbimortalidade das populações incluem doenças de transmissão respiratória, doenças transmitidas por insetos vetores, doenças relacionadas ao contato com coleções de água/solo e Infecções Sexualmente Transmissíveis (IST). Tais doenças mantêm relação direta com a pobreza e condições de vida inadequadas (SEGURADO AC, et al., 2016; SOUZA HP, et al., 2020).

O Brasil reduziu em $50 \%$ a proporção de óbitos associados às doenças infecciosas nos últimos trinta anos. Apesar do declínio, se mantém a frequência em um cenário de transição demográfica e epidemiológica caracterizado pela predominância concorrente de doenças transmissíveis e crônicodegenerativas, pelo agravamento daquelas consideradas controladas e eliminadas, como a emergência da Febre Amarela e Sarampo, e pelo contraste entre diferentes regiões do país. Além disso, têm ocorrido grandes epidemias virais por Chikungunya, Zika, Dengue, o vírus da imunodeficiência humana, e em mais evidência a pandemia pelo novo coronavírus (SARS-CoV-2), a qual tem afetado drasticamente o mundo todo (SOUZA HP, et al., 2020; LANA RM, et al., 2020; WALDMAN EA e SATO APS, 2016).

A participação hospitalar nas redes de vigilância epidemiológica já é bastante reconhecida, uma vez que os hospitais são a porta de entrada para as doenças. No entanto, isto envolve grandes recursos e desafios, como o acúmulo de dados, os quais nem sempre são analisados e transformados em informações que possam subsidiar tomadas de decisões. Ademais, poucos profissionais problematizam suas práticas e elaboram investigações regionais pertinentes quanto às políticas de saúde locais. Assim, faz-se necessário - aprimoramento da capacidade de investigação, além da utilização de métodos de estudos epidemiológicos para que se promova o embasamento científico e avanços na saúde (SOUZA HP, et al., 2020; LIMA KWS, et al., 2015).

Nesse sentido, pretende-se descrever dados clínico-epidemiológicos de Doenças de Notificação Compulsória (DNC) confirmadas e investigadas em um hospital universitário de referência na Amazônia Oriental Brasileira. 


\section{MÉTODOS}

Pesquisa descritiva, retrospectiva, transversal e de natureza quantitativa, realizado na Unidade de Vigilância em Saúde (UVS) de um hospital universitário referência em doenças infecto-parasitárias, situado em Belém - Pará, da Amazônia Oriental.

Amostra probabilística, incluindo notificações investigadas de pacientes internados no hospital, de janeiro a dezembro de 2018, ambos os sexos, todas as idades, procedentes de municípios do Pará segundo as mesorregiões do Marajó (MJ), Baixo Amazonas (BA), Metropolitana de Belém (MB), Nordeste Paraense (NP), Sudeste Paraense $\left(S_{E} P\right)$ e Sudoeste Paraense (SoP). Notificações investigadas de pacientes atendidos no ambulatório, ou notificações de funcionários, e aquelas com ausência de dados pertinentes à pesquisa, foram excluídas. As fichas de notificação foram lançadas no banco de dados, em uma planilha do programa Microsoft Office® Excel 2016.

As DNC investigadas de usuários foram classificadas em quatro grupos, onde o Grupo I (GI) se refere às doenças de transmissão respiratória: Coqueluche, Meningites, Hanseníase, Sarampo, Síndrome Respiratória Aguda Grave (SRAG), Tuberculose e Varicela; o Grupo II (GII) as doenças transmitidas por insetos vetores: Dengue, Doença de Chagas Aguda, Febre de Chikungunya, Leishmaniose Tegumentar Americana, Leishmaniose Visceral, Malária; o Grupo III (GIII) às doenças relacionadas ao contato com coleções de água/solo: Febre Tifóide, Leptospirose e Tétano Acidental; e o Grupo IV (GIV) às IST: Síndrome da Imunodeficiência Adquirida - Aids, Hepatites virais e Sífilis não especificada.

A coleta de dados ocorreu em outubro e novembro de 2019 após aprovação do Comitê de Ética sob o número 3.450.587, a partir de um formulário cujas variáveis foram: DNC, o mês da notificação, o critério diagnóstico considerado (laboratorial, clínico-epidemiológico, critério óbito), o município de procedência, faixa etária, sexo e o tipo de saída (melhorado, óbito). Estas foram categorizadas e agrupadas com o auxílio de filtros do programa Microsoft Office ${ }^{\circledR}$ Excel 2016, aplicando métodos estatísticos descritivos e inferenciais. Realizado análise descritiva, verificação de erros e inconsistências dos registros, correção e complementação dos dados.

A taxa de incidência foi calculada a partir do número de casos novos no município de procedência dividido pela população deste e multiplicado por 100 mil habitantes. A população de cada município foi de acordo com o último censo de 2010 do Instituto Brasileiro de Geografia e Estatística (IBGE). A taxa de mortalidade relacionada à DNC foi encontrada a partir do número de óbitos dos casos confirmados pelo total de casos confirmados, multiplicado por 100.

\section{RESULTADOS}

Notificados 2.755 DNC em 2018, considerando os critérios restaram 742 casos, dos quais $64,5 \%$ (478) eram por doenças de transmissão respiratória, 26,2\% (195) por IST, 6,6\% (49) transmitidas por insetos vetores e $2,7 \%$ (20) relacionadas ao contato com coleções de água/solo.

O Gráfico 1 evidencia Meningite (285) com maior quantitativo de casos do Gl, com destaque para meningite bacteriana não especificada (114), além da Tuberculose (176) na forma pulmonar (86). Vale ressaltar o ressurgimento do Sarampo com seis casos. No Gll, a Leishmaniose Visceral (31) teve grande evidência, acompanhada da Febre de Chikungunya (06) e Malária (06), sendo estes, do tipo Plasmodium vivax. Quanto ao GIII, a Leptospirose foi a mais notificada (11). No que se refere ao GIV, a Aids se destacou em $\geq 13$ anos (152), seguida da Sífilis não especificada (23) e Hepatites do tipo B (06) e C (12).

Considerando a periodicidade, as doenças se manifestaram principalmente de abril a setembro, sendo a Meningite (33\%), Febre de Chikungunya $(66,6 \%)$ e Malária $(33,3 \%)$ no $2^{\circ}$ trimestre; e a Tuberculose $(30,1 \%)$, Leishmaniose Visceral $(35,5 \%)$, Dengue (100\%) e Doença de Chagas $(66,7 \%)$ no $3^{\circ}$ trimestre do ano. Com exceção da Leptospirose $(63,6 \%)$ que predominou no $1^{\circ}$ trimestre e $33,3 \%$ de casos por malária no $4^{\circ}$ trimestre.

Em relação ao sexo, mais de $60 \%$ de usuários acometidos eram do sexo masculino, entretanto, no Gl houve majoritariedade feminina na Varicela Grave (100\%), Sarampo (66,6\%) e SRAG $(66,6 \%)$, bem como para Febre Tifoide (75\%) no grupo III. Coqueluche, Malária e Dengue não apresentaram diferença quanto aos sexos. 
Sobre a faixa etária, a Coqueluche (100\%) incidiu em sua maioria em menores de 01 ano, como SRAG (75\%). Febre de Chikungunya (33,3\%), Leishmaniose Visceral (74,3\%) e Febre Tifóide (75\%) manifestaramse na faixa de 01 a 09 anos. Os casos de Leptospirose variaram a partir de 10 anos. O GI e GIV prevaleceram entre as faixas de 20 a 29 anos, como a Meningite $(21,4 \%)$ e de 30 a 39 anos para casos de Tuberculose $(26,1 \%)$, Aids $\geq 13$ anos $(35,6 \%)$ e Sífilis não especificada $(34,8 \%)$, já no Tétano Acidental (40\%) eram de 50 a 59 anos.

O critério diagnóstico considerado foi o laboratorial, como confirmatório e definidor das DNC. No entanto, - Gl demonstrou que os casos de Coqueluche e Varicela grave consideraram apenas o critério clínico epidemiológico, como a Tuberculose mais de $50 \%$, e no Glll o Tétano acidental (80\%). Ressalta-se que sete casos de Aids $\geq 13$ anos foram considerados critério óbito. Para Hanseníase e Febre Tifoide não houve diferença quanto aos critérios.

Sobre o tipo de saída hospitalar dos usuários, a alta por melhora predominou nos grupos I, II e III. O grupo das IST's teve pouco menos da metade como óbitos (97), devido a $60 \%$ dos casos de Aids $\geq 13$ anos terem evoluído a óbito.

A taxa de incidência de DNC no estado do Pará foi de 9,79 casos/100 mil hab., onde $51,4 \%$ dos 144 municípios do estado houveram casos investigados e confirmados. A mesorregião maior em quantitativo de municípios (41/144) e, além disso, a mais incidente (466,6 casos/100 mil hab.) foi o NP. Das seis mesorregiões do estado, apenas no BA não houve casos notificados no hospital de referência (Gráfico 1).

Gráfico 1 - Doenças notificáveis investigadas e confirmadas por grupos, Pará, ano 2018.

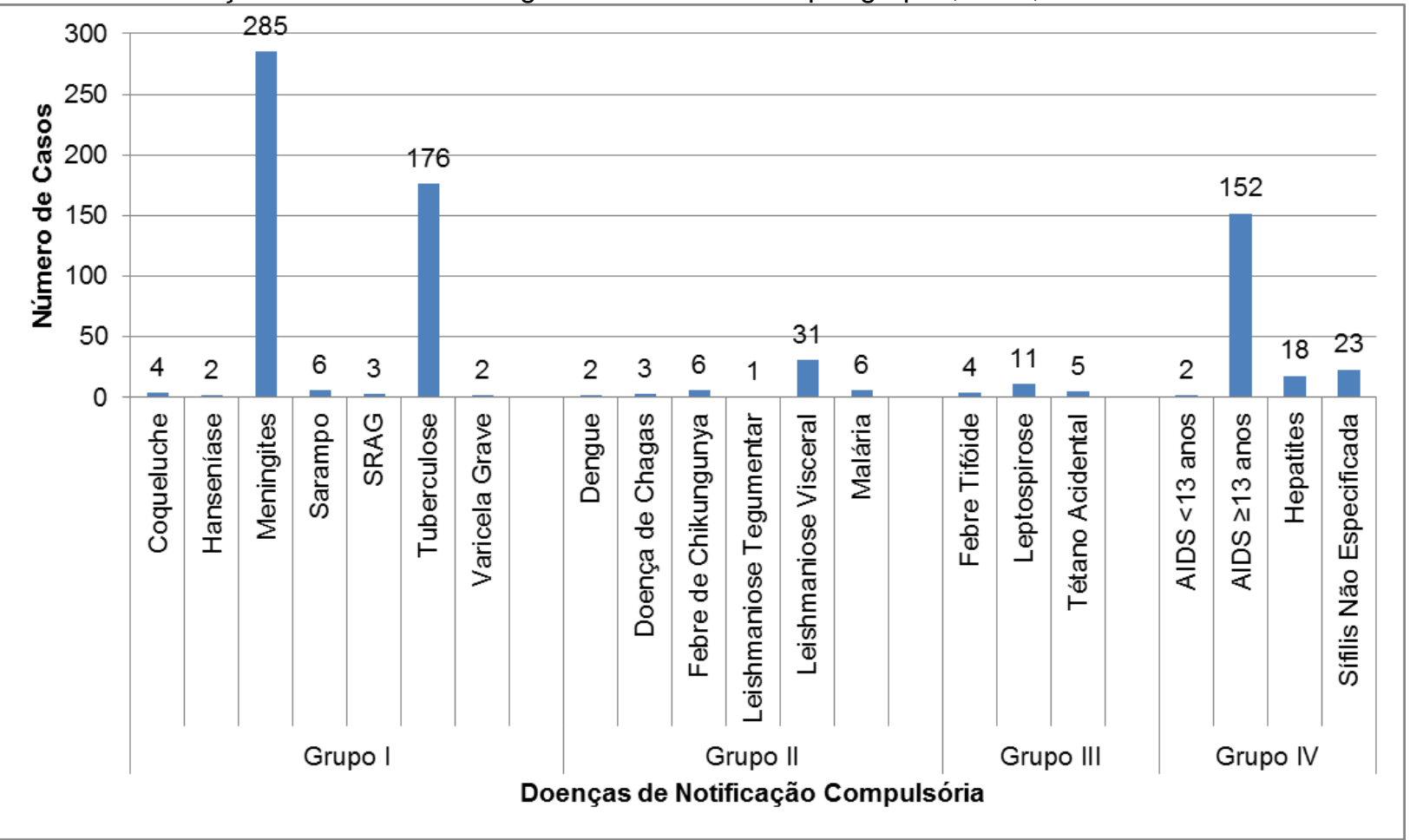

Fonte: Miranda VPB, et al., 2021.

No Gráfico 2 são ressaltadas apenas as maiores taxas de incidência e relevância epidemiológica das DNC no período estudado. A meningite foi uma das mais incidentes nas mesorregiões paraense, variando de 5,11 a 19,50 casos/100 mil hab. com exceção do SoP que teve registro por Malária do Gll com 7,09 casos em Jacareacanga e também notificados em Mocajuba no NP com 7,48 casos/ mil hab.

A Tuberculose do GI foi mais incidente em Santa Cruz do Arari (MJ) com 16,1 casos/100 mil hab. e em Santa Isabel do Pará (MB) com 10,17 casos/100 mil hab. A Leishmaniose Visceral do Gll predominou somente no NP, MJ e SEP no intervalo de 5,86 a 9,33 casos/ mil hab. 
O município de Primavera no NP teve destaque para as IST do GIV, apresentando casos de Aids $<13$ anos e Hepatites, com 9,74 casos/100 mil hab. para cada. As notificações por Aids $\geq 13$ anos sobressaiu nas três mesorregiões do MJ, NP e MB com 5,06, 7,28 e 7,97 casos/ 100 mil hab., respectivamente.

Destaque para os casos de Sarampo que ocorreram somente em Belém, com 0,43 casos/100 mil hab. E na mesorregião do NP tiveram outras DNC não menos comuns como Leptospirose (1,72 casos/100 mil hab.), Tétano Acidental (1,43 casos/100 mil hab.), Febre Tifóide (3,5 casos/100 mil hab.), Febre de Chikungunya (3,78 casos/100 mil hab.) e Doença de Chagas (3,24 casos/100 mil hab.).

No que concerne a taxa de mortalidade, a Aids ( $\geq 13$ anos) teve 12 óbitos/100 casos, sendo considerada a maior dentre as DNC estudadas, seguida pela Tuberculose (6 óbitos/100 casos), Meningite (4 óbitos/100 casos), Hepatites e Leishmaniose Visceral (0,5 óbitos/100 casos cada), Tétano Acidental (0,4 óbitos/100 casos), Sífilis não especificada (0,2 óbitos/100 casos), Varicela grave, Sarampo e Hanseníase (0,1 óbitos/100 casos cada) (Gráfico 2).

Gráfico 2 - Taxa de incidência das doenças notificáveis investigadas e confirmadas por grupos, segundo mesorregiões e seus respectivos municípios, Pará, ano 2018.

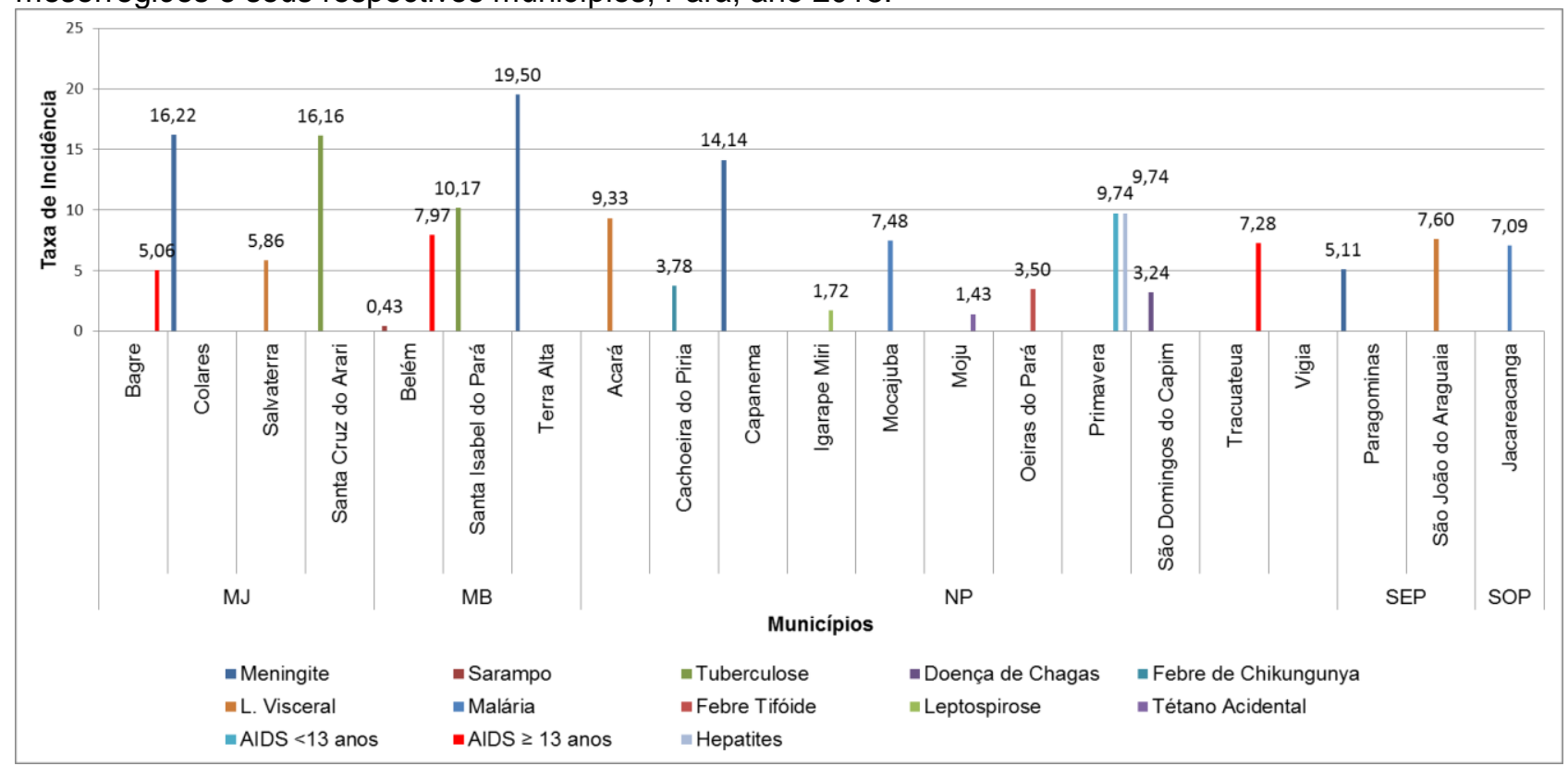

Fonte: Miranda VPB, et al., 2021.

\section{DISCUSSÃO}

Contato próximo entre infectados e suscetíveis, ocorrência de enchentes ou proliferação de insetos no ambiente, são algumas das condições comuns no cotidiano da população que facilitam a propagação de doenças (SEGURADO AC, et al., 2016). Filho EBS, et al. (2017) alega que as doenças de transmissão respiratória são muito frequentes e podem variar desde infecções agudas a mais graves, como as evidenciadas no estudo pela Meningite e Tuberculose.

No Brasil e no mundo a Meningite é considerada endêmica, com surtos periódicos em várias regiões, o Pará é responsável por 4,7\% das internações do setor de infectologia na Região Norte, além disso, Cruz $\mathrm{CM}$, et al. (2018) afirma que as estações outono/inverno colaboram para a transmissão, dado este confirmatório no estudo, com maior número de casos no $2^{\circ}$ trimestre do ano, considerado inverno paraense (DIAS FCF, et al. 2017).

Quanto à Tuberculose, o Brasil é um dos 22 países que concentram $80 \%$ da carga mundial desta doença (MINISTÉRIO DA SAÚDE, 2019a). Belém em 2018, estava entre as cinco capitais mais incidentes (62,7 casos/100 mil hab.), o que confirma neste estudo (MINISTÉRIO DA SAÚDE, 2020). Com relação à sazonalidade não se tem registro, apesar da pesquisa mostrar predominância no $3^{\circ}$ trimestre. 
Sobre os casos emergentes de Sarampo encontrados, estes corroboram com o achado de Silvério SMR (2019) com crescimento alarmante na região Norte, destaque para sete estados, três em estado de alerta e emergência na saúde pública contribuindo para a perda da certificação de erradicação, um retrocesso às conquistas alcançadas pela imunização.

As IST's representaram o segundo maior grupo de DNC deste achado e acarretam danos sociais, econômicos e sanitários para as populações, especialmente entre mulheres e crianças. A manifestação clínica devido à infecção pelo HIV ainda se mostra como um grave problema de saúde pública nos dias de hoje, constituindo uma miscelânea de epidemias regionais em sua quarta década (ABATI PAM e SEGURADO AC, 2015).

A região Norte do País apresenta tendência de crescimento das taxas de detecção dessa doença, corroborando com o presente resultado, o Pará ocupa o terceiro lugar a nível nacional pelos indicadores de taxas de detecção, mortalidade e primeira contagem de CD4 nos últimos cinco anos; e em relação às capitais, Belém está na primeira posição (MINISTÉRIO DA SAÚDE, 2019b). Os menores de 13 anos correspondem à transmissão vertical, modo de contaminação mais evidenciado em crianças a partir do final da década de 1990 (SPINARDI JR, et al., 2008).

A outra IST encontrada foi Hepatite dos tipos B e C. Conforme Nunes HM, et al. (2017), essa DNC é de distribuição mundial e seus tipos variam de frequência conforme a etiologia e as regiões geográficas, representando um grave problema de saúde pública mundial também. Sobre a Sífilis, informes oficiais da Organização Mundial da Saúde (OMS) indicam que, anualmente, ocorrem cerca de 12 milhões de novos casos na população adulta em todo o mundo, a maior parte em países em desenvolvimento (DANTAS LA, et al., 2017).

Em relação ao Gll, o desmatamento e as condições sanitárias inadequadas, aliados ao alto índice pluviométrico, estão entre os fatores que favorecem a transmissão de doenças por insetos vetores. As condições climáticas dos trópicos, associadas a variáveis políticas, econômicas e socioambientais também facilitam a manutenção do vetor (DINIZ PP, 2018). A Leishmaniose Visceral teve destaque, e segundo a OMS (2010), esta doença encontra-se entre as seis maiores endemias mundiais, afetando cerca de 2 milhões de pessoas a cada ano.

Sobre os casos de Febre de Chikungunya, desde 2014 a mesma é transmitida pelo Aedes aegypti no Brasil como uma das arboviroses que mais circulam no país (BRASIL, 2015). A Malária, considerada uma epidemia de alto risco, é atualmente ponderada à doença parasitária mais incidente do planeta. Segundo estimativas, a cada ano pode haver cerca de 250 milhões de casos novos e 880 mil mortes. Além disso, a exposição à infecção gira em torno de metade da população mundial, particularmente a população de países pobres (GRILO LM, et al., 2018). Outra arbovirose como a Dengue, apresenta-se como importante problema de saúde pública no Brasil, com uma elevada frequência de ciclos endêmicos (GONÇALVES CWB, et al., 2020).

No que tange às doenças do GIII, relevantes tanto em ambientes urbanos quanto rurais, a exposição acidental ocorre após contato com água ou lama. Destaca-se neste grupo, a Leptospirose, relacionada ao crescimento urbano desordenado e presença de lixo espalhado sobre vias e terrenos baldios, ambiente ideal para a proliferação da população de roedores, contribuindo para o aumento no número de casos. Ainda, este estudo reforça o que diz a literatura com a ocorrência dos casos no $1^{\circ}$ trimestre, que associam a ocorrência da doença a períodos de maiores índices pluviométricos e ao contato com água contaminada com urina de animais infectados (SEGURADO AC, et al., 2016; CARVALHO CBC, et al., 2017).

Em referência ao Tétano Acidental, tem-se observado um declínio no número de casos ao longo dos anos, com ocorrência em aposentados, pensionistas, entre trabalhadores agropecuários, da construção civil e domésticas, do mesmo modo para a Leptospirose. Diferente acerca da Febre Tifóide, considerada relevante nos países em desenvolvimento (MINISTÉRIO DA SAÚDE, 2017).

Neste estudo a maioria das DNC investigadas foram de usuários do sexo masculino, padrão conforme referências (LIMA KWS, et al., 2015; FILHO EBS, et al., 2017; DIAS FCF, et al., 2017; SILVÉRIO SMR, 2019; MINISTÉRIO DA SAÚDE, 2019b). Com relação à faixa etária adulta predominante, encontradas nos 
casos de Meningite, Tuberculose, Aids $\geq 13$ anos e Sífilis não especificada, corroboram com outros autores (DIAS FCF, et al., 2017; MINISTÉRIO DA SAÚDE, 2019a, 2019b; SANTOS BA, et al., 2015).

Em menores de 01 ano, Coqueluche e SRAG se destacaram devido à maior gravidade e a possibilidade de desenvolver complicações em lactentes (SANTOS BA, et al., 2015; MINISTÉRIO DA SAÚDE, 2018). Para a Leishmaniose Visceral, Silveira FT, et al. (2016) encontrou a mesma faixa etária infantil. Essa gravidade é reforçada em extremos de idade e presença de comorbidades (MINISTÉRIO DA SAÚDE, 2017; SILVEIRA FT, et al., 2016).

Quanto ao critério diagnóstico laboratorial, seguiu as recomendações de guias epidemiológicos, do contrário para Coqueluche que foi definida por clínico epidemiológico, apesar de se ter técnicas biomoleculares. Para a Tuberculose o diagnóstico clínico é considerado na impossibilidade de se comprovar por exames laboratoriais, tal como o Tétano Acidental, é válido o achado pelo estudo já que seu diagnóstico é essencialmente clínico. Em relação à varicela, exames laboratoriais são necessários para diagnóstico diferencial em casos graves (MINISTÉRIO DA SAÚDE, 2017; MINISTÉRIO DA SAÚDE, 2018).

Considerando a alta por melhora como tipo de saída, o Ministério da Saúde (2017) alega que o tratamento precoce é adequado por reduzir a letalidade das doenças e favorecer um prognóstico satisfatório, em contrapartida, a alta mortalidade em Aids $\geq 13$ anos, pode estar relacionada às infecções oportunistas, tumores, nefropatia e alterações neurológicas associadas, e à própria infecção pelo vírus (MINISTÉRIO DA SAÚDE, 2019b).

Dados referentes à temática em questão são bem escassos quando relacionados ao estado paraense. Se tratando de Amazônia Oriental, as doenças infecciosas e parasitárias nessas populações sempre foram objeto de debate, principalmente, quando relacionadas á questões ambientais e transformações socioeconômicas (MARINHO ACSM, et al., 2020).

As DNC mais incidentes que foram notificadas no hospital de referência da capital foram a Meningite, Tuberculose, Leishmaniose visceral e Aids se distribuindo nas cinco mesorregiões, com exceção do Baixo Amazonas, o que não descarta de haver casos atendidos nessa região no Hospital Regional em Santarém ou proximidades fora do estado, Amazonas. O estado do Pará, segundo dados do IBGE 2010, localizada na região Norte do Brasil com uma dimensão territorial de 1,2 milhões de $\mathrm{km}^{2}$ e uma densidade demográfica de $6,07 \mathrm{hab} / \mathrm{km}^{2}$, caracterizando como uma região geoeconômica menos populosa. Essa extensão territorial associada a difíceis acessos dificultariam o atendimento da população em serviços de referência.

Apesar dos dados notificados por meningite em 2018, segundo o Governo do Estado do Pará (2019), nenhum município destes teve registros de casos da doença meningocócica já nos primeiros meses de $2019 \mathrm{com}$ uma queda nas notificações. Para a Tuberculose, os municípios de procedência dos pacientes diferem dos encontrados por outros autores (MINISTÉRIO DA SAÚDE, 2019a; MINISTÉRIO DA SAÚDE, 2020). O alto número de Leishmaniose visceral coloca o estado como o terceiro maior em casos no Brasil (SILVEIRA FT, et al., 2016).

Consoante às mesorregiões, em ordem crescente de incidência a Aids $\geq 13$ anos destacou-se nos municípios de Belém, Tracuateua e Bagre. O Ministério da Saúde (2017) mostrou que no ranking dos 100 municípios mais incidentes do país, encontra-se Marituba em $3^{\mathrm{a}}$ lugar, seguido de Belém em $4^{\circ} \mathrm{e}$ Ananindeua está em 9o, com índices na população geral de 2014 a 2018 de 6,6, 6,5 e 6,3, respectivamente. Neste estudo, a capital também teve notoriedade, indicando a mais alta taxa desta DNC. Quanto à incidência para Aids <13 anos, o Ministério da Saúde (2019b) afirma que a maioria dos casos em 2018 $(86,2 \%)$ no país teve como via de infecção a transmissão vertical.

Dentre as DNC mais insidiosas no Gll está a Malária presente em Mocajuba no NP e única doença incidente no SoP, assumindo que o Pará é um dos estados mais endêmicos na Região Norte. A Febre de Chikungunya e Doença de Chagas tiveram certa notoriedade no NP, nos municípios de Cachoeira do Piriá e São Domingos, respectivamente. Diferente de outros autores, que registraram casos em Ananindeua, Abaetetuba Cametá e, principalmente, na capital Belém (MINISTÉRIO DA SAÚDE, 2015; SOUZA SB, et al., 2021). Estudos de incidência sobre Febre Tifóide, Leptospirose e Tétano Acidental são escassos no estado do Pará. 
Sobre a mortalidade, Aids $\geq 13$ anos, Tuberculose e Meningite tiveram as maiores taxas, valores que se refletem a nível regional com um aumento de $26 \%$ por Aids $\geq 13$ anos. Há registros de 4.534 óbitos por tuberculose no Brasil, com um coeficiente de mortalidade de 2,2 óbitos/100 mil hab., sendo o Pará alocado entre os quatro estados com maior coeficiente de mortalidade (3,0 óbitos/100 mil hab.). Para Meningite, o Governo do Estado do Pará apresenta registros de redução no coeficiente de mortalidade no estado com 0,11 óbitos/100 mil hab. no período de janeiro a abril de 2019 contra 0,16 óbitos/100 mil hab. no mesmo período do ano passado (SECRETARIA DE ESTADO DA SAÚDE DO PARÁ, 2019; MINISTÉRIO DA SAÚDE, 2019b; MINISTÉRIO DA SAÚDE, 2017).

\section{CONCLUSÃO}

Os dados apresentados neste estudo nos alertam sobre as Doenças de Notificação Compulsória de destaque no estado do Pará, como aquelas por transmissão respiratória mais evidente em períodos chuvosos, bem como o perfil no sexo masculino com destaque para lactentes, crianças e adultos jovens, confirmadas por critério laboratorial. Municípios do Nordeste Paraense tiveram grande visibilidade neste estudo, provavelmente devido à relação com fluxos migratórios, desmatamentos e impacto com as relações socioambientais que talvez justifiquem ênfase a altas incidências desses municípios. Em razão do baixo número de estudos epidemiológicos na Amazônia Oriental, especificamente no estado do Pará, faz-se necessário que mais pesquisas nesse contexto sejam produzidas, e que estas sejam cada vez mais relacionadas à região e sua população, podendo servir como indicador de desenvolvimento e nortear a construção e/ou aprimorar políticas públicas de saúde que se moldem às reais necessidades da população paraense.

\section{AGRADECIMENTOS}

Pelo rico espaço de aprendizagens e pesquisa, às residências multiprofissionais em saúde e a todas as instituições que colaboram e oportunizam os profissionais residentes no estado do Pará. Pelo apoio e colaboração, à Terezinha de Bastiani, enfermeira aposentada da Unidade de Vigilância Epidemiológica.

\section{REFERÊNCIAS}

1. ABATI PAM, SEGURADO AC. Testagem anti-HIV e estágio clínico na admissão de indivíduos em serviço de saúde especializado. Pará, Brasil. Revista de Saúde Pública, 2015; 49: 1-8.

2. CARVALHO CBC, et al. Leptospirose humana no estado do Rio de Janeiro: análise espaço-temporal e perfil dos casos confirmados no período de 2007 a 2014. Acad Rev Cient da Saúde, 2017; 2(3): 10-22.

3. CRUZ CM, et al. Incidência de meningite relacionada às condições sazonais no município de Maceió entre 2007 e 2017. Caderno de Graduação-Ciências Biológicas e da Saúde-UNIT-ALAGOAS, 2018; 5(1): 205.

4. DANTAS LA, et al. Perfil epidemiológico de sífilis adquirida diagnosticada e notificada em hospital universitário materno infantil. Rev Enfermeria Global, 2017; 16(46): 237-245.

5. DIAS FCF, et al. Meningite: aspectos epidemiológicos da doença na região norte do Brasil. Revista de Patologia do Tocantins, 2017; 4(2): 46-49.

6. DINIZ PP. Vigilância entomológica de vetores de arbovírus na cidade de São Paulo: análise espaço temporal de criadouros, de acordo com fatores sazonais e socioeconômicos, no período de 2012 a 2016 [Tese de doutorado]. São Paulo: Faculdade de Saúde Pública da Universidade de São Paulo (USP), 2018.

7. FILHO EBS, et al. Infecções Respiratórias de Importância Clínica: uma Revisão Sistemática. REVISTA FIMCA, 2017; 4:1.

8. GONÇALVES CWB, et al. Estudo Epidemiológico da Dengue em um Estado do Norte do Brasil. Amazônia: Science \& Health, 2020; 8(3): 83-90.

9. GOVERNO DO ESTADO DO PARÁ. Cai número de casos e óbitos por meningite no Pará. Belém: Secretaria de Estado de Saúde Pública, 2019. Disponível em: http://www.saude.pa.gov.br/2019/04/23/cai-numero-de-casos-eobitos-por-meningite-no-para/. Acesso em: 4 de novembro de 2019.

10. GRILO LM, et al. A geografia da malária na região norte do Brasil entre 2003 e 2016. In: Anais do Encontro Acadêmico de Engenharia Ambiental. 2018 out 12-15; Lorena. São Paulo: ENAMB, 2018; p. 18.

11. LANA RM, et al. Emergência do novo coronavírus (SARS-CoV-2) e o papel de uma vigilância nacional em saúde oportuna e efetiva. Cadernos de Saúde Pública, 2020; 36: e00019620. 
12. LIMA KWS, et al. Percepção dos gestores sobre o uso de indicadores nos serviços de saúde. Saúde social, 2015; 24(1): 61-71.

13. MARINHO ACSM, et al. Saúde ambiental e doenças diarreicas: indicadores socioeconômicos, ambientais e sanitários em um município amazônico. Research, Society and Development, 2020; 9 (9): e659997803-e659997803.

14. MINISTÉRIO DA SAÚDE. Portaria no 264, de 17 de fevereiro de 2020. Altera a Portaria de Consolidação no 4/GM/MS, de 28 de setembro de 2017, para incluir a doença de Chagas crônica, na Lista Nacional de Notificação Compulsória de doenças, agravos e eventos de saúde pública nos serviços de saúde públicos e privados em todo o território nacional. Diário Oficial da União, Brasília - DF, Brasil, 2020. Disponível em: https://bvsms.saude.gov.br/bvs/saudelegis/gm/2020/prt0264_19_02_2020.html. Acesso em: 19 de novembro de 2020.

15. MINISTÉRIO DA SAÚDE. Secretaria de Vigilância em Saúde. Departamento de Vigilância Epidemiológica. Brasil Livre da Tuberculose: evolução dos cenários epidemiológicos e operacionais da doença. Boletim Epidemiológico. Brasília - DF, Brasil, 2019a; 50. Disponível em: https://portalarquivos2.saude.gov.br/images/pdf/2019/marco/22/2019009.pdf. Acesso em: 27 de janeiro de 2021.

16. MINISTÉRIO DA SAÚDE. Secretaria de Vigilância em Saúde. Departamento de Vigilância Epidemiológica. Boletim epidemiológico. Brasília - DF, Brasil, 2020. Disponível em: https://www.saude.gov.br/images/pdf/2020/marco/24/Boletim-tuberculose-2020-marcas--1-.pdf. Acesso em: 7 de abril de 2020.

17. MINISTÉRIO DA SAÚDE. Secretaria de Vigilância em Saúde. Departamento de Vigilância Epidemiológica. Boletim Epidemiológico o 32. Brasília - DF, Brasil, 2018. Disponível em: https://portalarquivos2.saude.gov.br/images/pdf/2018/marco/26/2018-009.pdf. Acesso em: 20 de abril de 2019.

18. MINISTÉRIO DA SAÚDE. Secretaria de Vigilância em Saúde. Departamento de Vigilância Epidemiológica. Boletim Epidemiológico, HIV/Aids - 2019. Brasil, 2019b. Disponível em: http://www.aids.gov.br/pt-br/pub/2019/boletimepidemiologico-de-hivaids-2019. Acesso em: 13 de março de 2020.

19. MINISTÉRIO DA SAÚDE. Secretaria de Vigilância em Saúde. Departamento de Vigilância Epidemiológica. Guia de vigilância em Saúde. 2. ed. Brasília - DF, Brasil, $2017 . \quad$ Disponível em: https://portalarquivos.saude.gov.br/images/pdf/2017/outubro/06/Volume-Unico-2017.pdf. Acesso em: 20 de abril de 2019.

20. MINISTÉRIO DA SAÚDE. Secretaria de Vigilância em Saúde. Secretaria de Atenção Básica. Febre de chikungunya: manejo clínico. Brasília - DF, Brasil, $2015 . \quad$ Disponível em: https://bvsms.saude.gov.br/bvs/publicacoes/chikungunya_manejo_clinico.pdf. Acesso em: 28 de agosto de 2019.

21. NUNES HM, et al. As hepatites virais: aspectos epidemiológicos, clínicos e de prevenção em municípios da Microrregião de Parauapebas, sudeste do estado do Pará, Brasil. Revista Pan-Amazônica de Saúde, 2017; 8(2): 7-7.

22. OMS. Controle das leishmanioses: relatório de uma reunião do Comitê de Especialistas da OMS no Controle das Leishmanioses. Genebra: Organização Mundial da Saúde, 2010; 22-26p. Disponível em: https://apps.who.int/iris/handle/10665/44412. Acesso em: 27 de agosto de 2019.

23. SANTOS BA, et al. Coqueluche em crianças menores de 3 anos de idade hospitalizadas em 2011 e 2012 . Clinical and Biomedical Research, 2015; 35(4): 227-232.

24. SEGURADO AC, et al. Saúde nas metrópoles - Doenças infecciosas. Estudos avançados, 2016; 30(86): $29-49$.

25. SILVEIRA FT, et al. Revendo a trajetória da leishmaniose visceral americana na Amazônia, Brasil: de Evandro Chagas aos dias atuais. Revista Pan-Amazônica de Saúde, 2016; 7: 15-22.

26. SILVÉRIO SMR. Perfil epidemiológico do Sarampo na região norte brasileira no ano de 2018 [Trabalho de Conclusão de Curso]. Brasília (DF): Faculdade de Ciências da Educação e Saúde, Centro Universitário de Brasília, 2019.

27. SOUZA HP, et al. Doenças infecciosas e parasitárias no Brasil de 2010 a 2017: aspectos para vigilância em saúde. Revista Panamericana Salud Publica, 2020; 44: e10.

28. SOUZA SB, et al. Perfil epidemiológico da doença de Chagas aguda na região norte do Brasil no ano de 2015-2019. Revista Eletrônica Acervo Saúde, 2021; 13(7): e8200.

29. SPINARDI JR, et al. Adolescer com HIV: saber, conhecer e conviver. Revista adolescência e saúde, 2008; 4(2).

30. WALDMAN EA, SATO APS. Trajetória das doenças infecciosas no Brasil nos últimos 50 anos: um contínuo desafio. Revista de Saúde Pública, 2016; 50-68. 